\title{
Molecular Survey of Tick-Borne
} Pathogens Reveals Diversity and Novel Organisms With Veterinary and Public Health Significance in Wildlife From a National Nature Reserve of China Jifei Yang ${ }^{1 *}$, Xiaojun Wang ${ }^{2}$, Jinming Wang ${ }^{1}$, Zhijie Liu ${ }^{1}$, Qingli Niu ${ }^{1}$,
Muhammad Uzair Mukhtar ${ }^{1}$, Guiquan Guan ${ }^{1}$ and Hong Yin ${ }^{1,3 *}$

\section{OPEN ACCESS}

Edited by:

Giulia Morganti,

University of Perugia, Italy

Reviewed by:

Münir Aktaş,

Firat University, Turkey

Alessandra Cafiso,

University of Milan, Italy

*Correspondence:

Jifei Yang

yangjifei@caas.cn

Hong Yin

yinhong@caas.cn

Specialty section:

This article was submitted to

Parasitology,

a section of the journal

Frontiers in Veterinary Science

Received: 23 March 2021 Accepted: 10 June 2021 Published: 12 July 2021

Citation:

Yang J, Wang X, Wang J, Liu Z, Niu Q, Mukhtar MU, Guan $G$ and Yin $H$ (2021) Molecular Survey of Tick-Borne Pathogens Reveals Diversity and Novel Organisms With Veterinary and Public Health Significance in Wildlife From a National Nature Reserve of China. Front. Vet. Sci. 8:682963. doi: 10.3389/fvets.2021.682963

\begin{abstract}
${ }^{1}$ State Key Laboratory of Veterinary Etiological Biology, Key Laboratory of Veterinary Parasitology of Gansu Province, Lanzhou Veterinary Research Institute, Chinese Academy of Agricultural Sciences, Lanzhou, China, ${ }^{2}$ Tangjiahe National Nature Reserve, Qingchuan, China, ${ }^{3}$ Jiangsu Co-innovation Center for Prevention and Control of Important Animal Infectious Diseases and Zoonoses, Yangzhou, China
\end{abstract}

Wildlife is involved in the maintenance and transmission of various tick-borne pathogens. The objective of the present study was to determine the occurrence and diversity of tick-borne pathogens in free-ranging wild animals collected from Tangjiahe National Nature Reserve of China. Blood or liver samples from 13 wild animals (5 takin, 3 Himalayan goral, 3 Reeves' muntjac, 1 forest musk deer, and 1 wild boar) were collected and screened for piroplasm, Anaplasma spp., Ehrlichia spp., and spotted fever group (SFG) rickettsiae by PCR-based on different gene loci. Three Theileria species, a potential novel Theileria parasite (Theileria sp. T4) and two Anaplasma species were identified in those wildlife. Theileria capreoli was found in Himalayan goral, Reeves' muntjac, and forest musk deer; Theileria luwenshuni, Theileria uilenbergi, and a potential novel, Theileria parasite (Theileria sp. T4), were identified in takin. Meanwhile, Anaplasma bovis was identified in Himalayan goral, takin, Reeves' muntjac, forest musk deer, and wild boar; Anaplasma phagocytophilum and related strains was found in takin, Reeves' muntjac, and forest musk deer. All wildlife included in this study was negative for Babesia, Anaplasma ovis, Anaplasma marginale, Ehrlichia, and SFG rickettsiae. Moreover, coinfection involving Theileria spp. and Anaplasma spp. was observed in eight wild animals. This study provided the first evidence of tick-borne pathogens in free-ranging wild animals from the nature reserve, where contact between domestic and wild animals rarely occurs.

Keywords: tick-borne pathogen, Theileria, Anaplasma bovis, Anaplasma phagocytophilum, wildlife, nature reserve

\section{INTRODUCTION}

Tick-borne diseases have been becoming increasingly important with the increase in incidences and geographic distribution (1). They cause extensive economic losses in the livestock industry and also impose a growing health burden on human populations worldwide. In nature, the causative agents are maintained in complex transmission cycles involving ticks, various domestic, and wild animals 
(2). In the past three decades, an increasing number of new species, genotypes, or genetic variants of tick-borne pathogens were identified in ticks and domestic and wild animals, some of which have been subsequently recognized as human pathogens, such as Anaplasma phagocytophilum, Candidatus Neoehrlichia mikurensis, Rickettsia slovaca, Rickettsia raoultii, Rickettsia conorii, Babesia venatorum, and Babesia divergens (3). The global impact of tick-borne diseases on livestock, along with their zoonotic potential, has attracted great attention and interest in the surveillance for ticks and their associated pathogens not only in domestic but also in wild animals.

Wild animals serving as reservoir hosts for various tickborne infections has been well-established globally. They play a critical role in the maintenance of the endemic cycle of tickborne diseases, which increase the risk of disease transmission to humans and domestic animals due to their movements potentially affect the pathogens and vectors distribution (3). Therefore, the identification of the wild host species that serve as important reservoirs of tick-borne infections is essential. However, limited information is available on tickborne pathogens in wild animals when compared with ticks and domestic animals in China and remains to be disclosed, especially in those from nature reserves, where contact between wild and domestic animals rarely occurs. Those animals fulfill an important mission in the persistence of tick vectors and pathogens in nature (4). In the present study, the occurrence and diversity of tick-borne agents with veterinary and medical significance were investigated in wild animals collected from Tangjiahe National Nature Reserve of China, which is a natural habitat for many endangered and national protected wildlife.

\section{MATERIALS AND METHODS}

\section{Study Site and Collection of Specimens}

The Tangjiahe National Nature Reserve is a protected area located in Qingchuan County of Sichuan Province, southwestern China. The reserve was established in 1978 and dedicated to protecting the habitat and wildlife. Occupying an area of about 40,000 ha, 481 species of vertebrates inhabited in this reserve, including 72 national key protected wildlife. This area is one of the hotspot areas for global biodiversity conservation and is also an important habitat of many endangered wildlife, such as giant pandas, golden monkeys, and takins.

From March 2016 to April 2017, 13 dead wild animals were found in Tangjiahe National Nature Reserve, including takin (Budorcas taxicolor, class I national protected species, $n$ $=5$ ), forest musk deer (Moschus berezovskii, class I national protected species, $n=1$ ), Himalayan goral (Naemorhedus goral, class II national protected species, $n=3$ ), Reeves' muntjac (Muntiacus reevesi, $n=3$ ), and wild boar (Sus scrofa, $n=1$ ). The ethylenediaminetetraacetic acid (EDTA) blood or liver samples were collected from those animals, and DNA was isolated from $200 \mu \mathrm{l}$ whole blood or $25 \mathrm{mg}$ liver by using QIAamp DNA Mini Kit according to manufacturer's instructions. The concentration of DNA samples $(\mathrm{ng} / \mu \mathrm{l})$ was measured by using NanoDrop2000 (Thermo Scientific, Waltham, MA, USA).

\section{PCR Reactions}

Polymerase chain reactions were carried out to screen the samples for detection of $18 \mathrm{~S}$ rRNA sequences of piroplasm; $16 \mathrm{~S}$ rRNA sequences of A. phagocytophilum, Anaplasma bovis, and Ehrlichia spp.; msp4 sequences of Anaplasma marginale and Anaplasma ovis; and ompA sequences of spotted fever group (SFG) rickettsiae, according to previously described (Table 1). The PCR amplifications were performed in an automatic thermocycler (Bio-Rad, Hercules, CA, USA) with a final volume of $25 \mu$ l containing $2.5 \mu \mathrm{l}$ of $10 \times$ PCR buffer $\left(\mathrm{Mg}^{2+}\right.$ Plus), $2.0 \mu \mathrm{l}$ of each deoxyribonucleotide triphosphate $(\mathrm{dNTP})$ at $2.5 \mathrm{mM}, 1.0 \mu \mathrm{l}$ of each primer (10 pmol), $2.0 \mu \mathrm{l}$ of DNA samples (blood DNA, 18.4$24.3 \mathrm{ng} / \mu \mathrm{l}$; liver DNA, 94.8-134.2 $\mathrm{ng} / \mu \mathrm{l}), 1.25 \mathrm{U}$ of Taq DNA polymerase (TaKaRa, Dalian, China), and 16.25 $\mu \mathrm{l}$ of distilled water. Positive (containing DNA of the corresponding organisms) and negative controls were included in all amplifications. PCR products were analyzed by agarose gel electrophoresis (1.2\%) and visualized under UV transillumination after Goldview staining (Solarbio, Beijing, China).

\section{DNA Sequencing and Phylogenetic Analysis}

The positive amplicons were purified from agarose gel with the AxyPrepTM DNA Gel Extraction Kit (Axygen, Union City, CA, USA) according to the manufacturer's instructions. Purified products were cloned into pGEM-T Easy vector (Promega, Madison, WI, USA) and transformed into Escherichia coli JM109 competent cells (TaKaRa). Three clones were selected randomly for sequencing (GenScript, Nanjing, China). The obtained sequences were compared with those deposited in GenBank using BLASTn, and multiple-sequence alignments were constructed by using ClustalW algorithm in the MegAlign program of the Lasergene software (DNASTAR, Madison, WI, USA). Phylogenetic relationships were assessed by using neighbor-joining (NJ) method with the Kimura twoparameter model, and the bootstrap test was replicated 1,000 times (12).

\section{Compliance With Ethical Standards}

Animal treatments and sample preparation complied with the Animal Ethics Procedures and Guidelines and was approved by the Animal Ethics Committee of Lanzhou Veterinary Research Institute, Chinese Academy of Agricultural Sciences.

\section{Nucleotide Sequence Accession Numbers}

The nucleotide sequences obtained in this study were deposited in the GenBank database and designated accession numbers: MH179333 for 18S rRNA gene sequence of Theileria uilenbergi, MH179334 and MH179335 for 18S rRNA gene sequences of Theileria capreoli, MH179336 for 18S rRNA gene sequence of Theileria luwenshuni, MH179337 for 18S rRNA gene sequence of Theileria sp. T4; MH180816 and MH180817 for 16S rRNA gene sequences of $A$. bovis, and MH180818-MH180820 for 16S rRNA gene sequences of $A$. phagocytophilum. 
TABLE 1 | Primers and PCR amplification conditions.

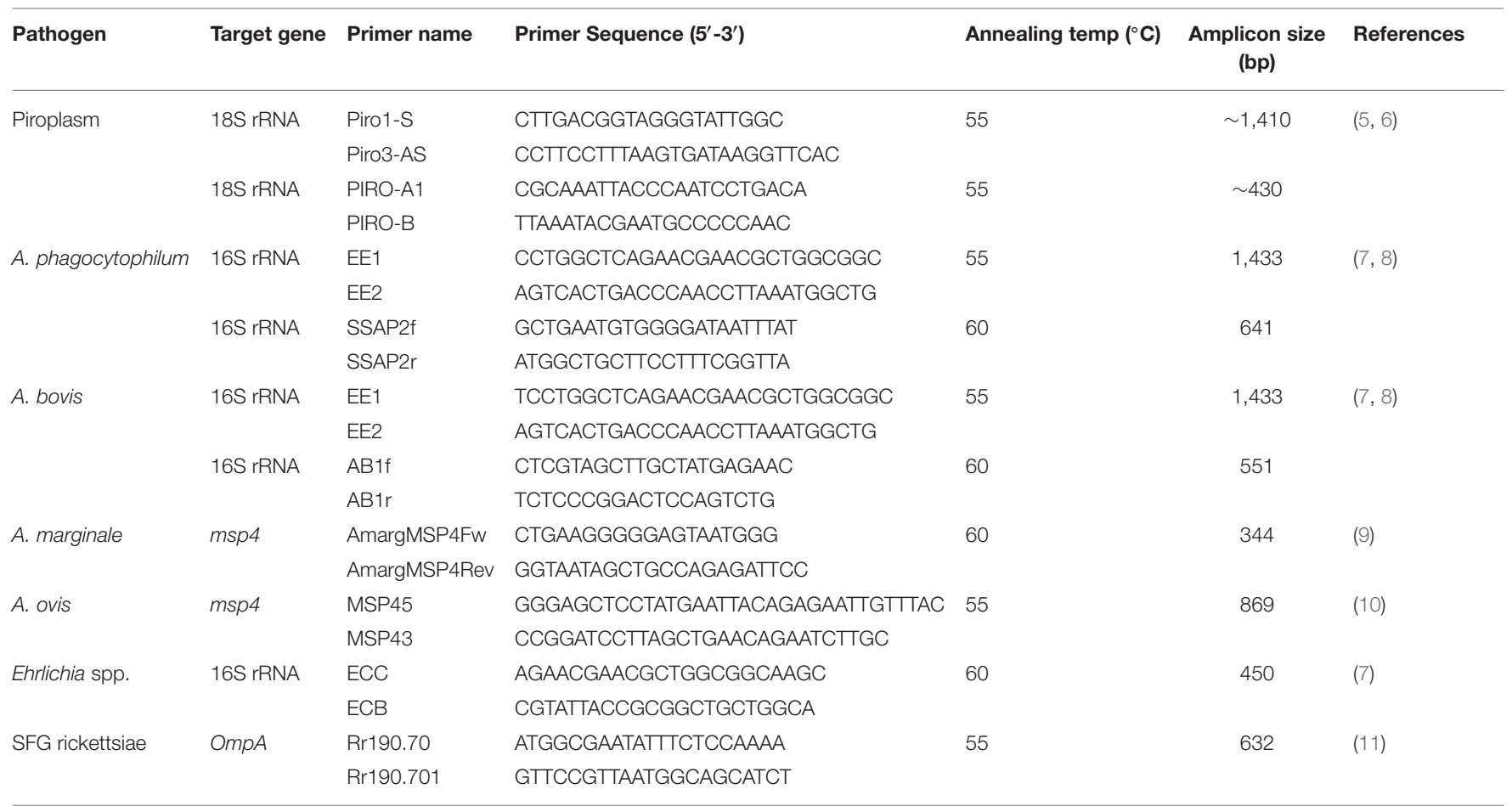

TABLE 2 | The piroplasm and Anaplasma spp. identified in wildlife from Tangjiahe National Nature Reserve, China.

\begin{tabular}{|c|c|c|c|c|c|}
\hline Animal species & Scientific name & Sample & Piroplasm & A. bovis & A. phagocytophilum \\
\hline Himalayan goral & Naemorhedus goral & Blood & T. capreoli & + & - \\
\hline Himalayan goral & Naemorhedus goral & Liver & - & - & - \\
\hline Himalayan goral & Naemorhedus goral & Liver & - & - & - \\
\hline Takin & Budorcas taxicolor & Blood & Theileria sp. T4 & + & + \\
\hline Takin & Budorcas taxicolor & Blood & T. uilenbergi & + & + \\
\hline Takin & Budorcas taxicolor & Liver & T. luwenshuni & - & - \\
\hline Reeves' muntjac & Muntiacus reevesi & Blood & - & + & + \\
\hline Reeves' muntjac & Muntiacus reevesi & Blood & T. capreoli & + & - \\
\hline Reeves' muntjac & Muntiacus reevesi & Liver & T. capreoli & + & - \\
\hline Forest musk deer & Moschus berezovskii & Blood & T. capreoli & + & + \\
\hline
\end{tabular}

\section{RESULTS}

In the present study, piroplasms, $A$. bovis and $A$. phagocytophilum, were detected in 9,10 , and 4 of 13 wildlife included in this study (Table 2). The partial 18S rRNA gene sequences ( $\sim 430 \mathrm{bp})$ of piroplasm (Babesia/Theileria) were amplified, and sequence analysis revealed three Theileria species and a potential novel Theileria parasite in those wildlife. No Babesia sequence was found in those amplicons. Theileria capreoli was found in one Himalayan goral, two Reeves' muntjac, and one forest musk deer (Table 2). The 18S rRNA sequences of T. capreoli from Himalayan goral showed $98.7 \%$ identity with T. capreoli isolate Qilian-Mountain 13 (KJ188219) (13); the isolates from Reeves' muntjac and forest musk deer showed 100\% identity with $T$. capreoli isolate $8 \mathrm{P}$ (KJ451469) (5). Theileria luwenshuni was identified in three takins, and all three isolates were $100 \%$ identical to the T. luwenshuni isolate Dawu B5 (JX469514) (14). Theileria uilenbergi (P6-a, MH179333) was found in one takin; it was $99.7 \%$ identical to the T. uilenbergi isolate Li 2 (JF719835). A potential novel Theileria parasite (Theileria sp. T4) identified in one takin showed $99.7 \%$ identity with the Theileria sp. Iwate 2-2 (AB602881).

To further verify the Theileria species identified in this study, $\sim 1,410$-bp fragments for $18 \mathrm{~S}$ RNA of T. capreoli, T. luwenshuni, 
and Theileria sp. T4 were amplified, whereas no amplicons were detected for T. uilenbergi (P6-a, MH179333). Sequence and phylogenetic analysis revealed that the T. capreoli isolates (Pb2-a, MH179334; Pb11-a, MH179335) were clustered together with the isolates from Reeves' muntjac (KJ451469), red deer (KJ188219), European roe deer (AY276011), and white-lipped deer (JX134576) with 99.0-99.7\% sequence identity; Theileria sp. T4 (MH179337) was closely related to the strains CC3A (AB012201) and Iwate (AB602881) of an unclassified Theileria species detected from Capricornis crispus and Japanese serows with 99.9\% sequence identity, respectively; T. luwenshuni (Pb7a, MH179336) was closely related to the T. luwenshuni isolate 2 (JF719832), Dawu B5 (JX469514), Li 6 (JF719833), and Iwate 1-1 (AB602877) with 100\% sequence identity (Figure 1).

Anaplasma bovis was detected in one Himalayan goral, four takin, three Reeves' muntjac, one forest musk deer, and one wild boar (Table 2). The $16 \mathrm{~S}$ rRNA sequences of $A$. bovis obtained from positive samples were $99.6-100 \%$ identical to each other. Three isolates (Ab4a, MH180817) from two takins and one forest musk deer were $100 \%$ identical to the $A$. bovis isolates KWDAB2 (GU556627), 2-44Ab (KF465981), ABKGHL (AF470698), C_AP_1-2 (LC068731), and ES1 (KC811530) derived from Korean water deer, Reeves' muntjac, Haemaphysalis longicornis, sika deer, and Elephantulus myurus, respectively. The remaining isolates (Ab2a, MH180816) were 100\% identical to A. bovis isolates b1 (KX115423), b2-25a (MF066914), Ab3a (KJ639883), NX1-1 (KM186945), T-KOAB1 (KC311344), giraffe 2013-5 (KU870666), and JC4-4 (KM186944) from cattle, sheep, red deer, roe deer, H. longicornis, giraffe, and Procapra gutturosa, respectively.

Anaplasma phagocytophilum infection was found in two takins, one Reeves' muntjac, and one forest musk deer (Table 2). Three 16S RNA sequence variants of A. phagocytophilum were identified that shared $98.5-99.8 \%$ sequence similarity. The $16 \mathrm{~S}$ RNA sequence of variant 1 (Ap4a, MH180818) and variant 2 (Ap5-b, MH180819) detected from takin showed 99.8-100\% identity with $A$. phagocytophilum-like 1 strain derived from cattle (KX702974) from Tunisia (15). The variant 3 (Ap11-b, MH180820) identified from Reeves' muntjac and forest musk deer was $100 \%$ identical to the A. phagocytophilum isolate detected from white yaks (KT824826).

All wildlife included in this study were negative for A. ovis, A. marginale, Ehrlichia spp., and SFG rickettsiae. The coinfection of Theileria spp. and Anaplasma spp. was found in eight wildlife, including one Himalayan goral, four takins, two Reeves' muntjac, and one forest musk deer; the coinfection of two Anaplasma species (A. bovis and A. phagocytophilum) was observed in two takins, one Reeves' muntjac, and one forest musk deer (Table 2).

\section{DISCUSSION}

Wild animals carry various tick species and act as important reservoir hosts for many tick-borne pathogens. The present study investigated tick-borne bacteria and protozoan parasites, and three Theileria (T. capreoli, T. uilenbergi, and T. luwenshuni), a potential novel Theileria parasite (Theileria sp. T4), and two Anaplasma species (A. bovis, A. phagocytophilum, and related strains) were identified in wildlife from Tangjiahe National Nature Reserve situated in Sichuan Province, southwestern China.

Theileria is tick-borne protozoa, infecting both leukocytes and erythrocytes of their vertebrate hosts (16). Domestic and wild animals are susceptible to different Theileria species, many of which are previously known to be reasonably host specific. However, increasing evidence showed that some Theileria species have been identified in unexpected hosts from different geographic locations, especially in wild animals. In this study, T. capreoli was identified in Himalayan goral, Reeves' muntjac, and forest musk deer. This organism is initially described from roe deer and is considered to be a benign parasite of wild cervids (17); the infections have also been recorded in fallow deer, red deer, and Chinese water deer $(17,18)$. Theileria uilenbergi and T. luwenshuni are considered to be highly pathogenic parasites of small domestic ruminants (19). The occurrence of these two Theileria species has been widely reported in sheep and goats from China and causes great economic losses for the livestock industry (19). However, there are sporadic reports of $T$. uilenbergi and $T$. luwenshuni in wild animals. To date, infection of $T$. luwenshuni (Theileria sp. China 1) was observed in a few Chinese water deer and roe deer from South Korea, roe deer, sika deer, and red deer from China $(18,20,21)$, while T. uilenbergi has been detected in red deer, sika deer, and Reeves' muntjac from China $(5,13)$. The present study constitutes the first evidence of T. luwenshuni and T. uilenbergi infections in takins. As mentioned above, these two Theileria parasites are high pathogenic for sheep and goats; however, their pathogenicity for wild animals is still unclear, which warrants further investigation. Moreover, a potential novel Theileria parasite (Theileria sp. T4) was identified in takin from this study that has been previously reported in Capricornis crispus (AB012201) and Japanese serows (AB602881).

The members in the genus Anaplasma are obligate intracellular bacteria that replicate in various cell types (22). These agents are associated with infections in humans and many species of domestic and wild animals. Of those well-known Anaplasma species, A. bovis and A. phagocytophilum infect a wide range of both domestic and wild animals, whereas the remaining Anaplasma species are relatively host specific (23). Since A. bovis was first described in cattle, the infection has also been recognized in other ruminant and non-ruminant animals, such as sheep, goats, various deer, Mongolian gazelle, wild boars, dogs, raccoons, and cotton-tail rabbits, (24-26). In the present study, A. bovis was detected in all five wild animal species investigated from Tangjiahe National Nature Reserve. These results indicated that Himalayan goral, takin, Reeves' muntjac, forest musk deer, and wild boar can act as reservoir hosts for A. bovis.

Anaplasma phagocytophilum is a well-known intragranulocytic bacteria with medical and veterinary importance worldwide. The host range of this organism is wide, including rodents, ruminants, carnivores, birds, and 


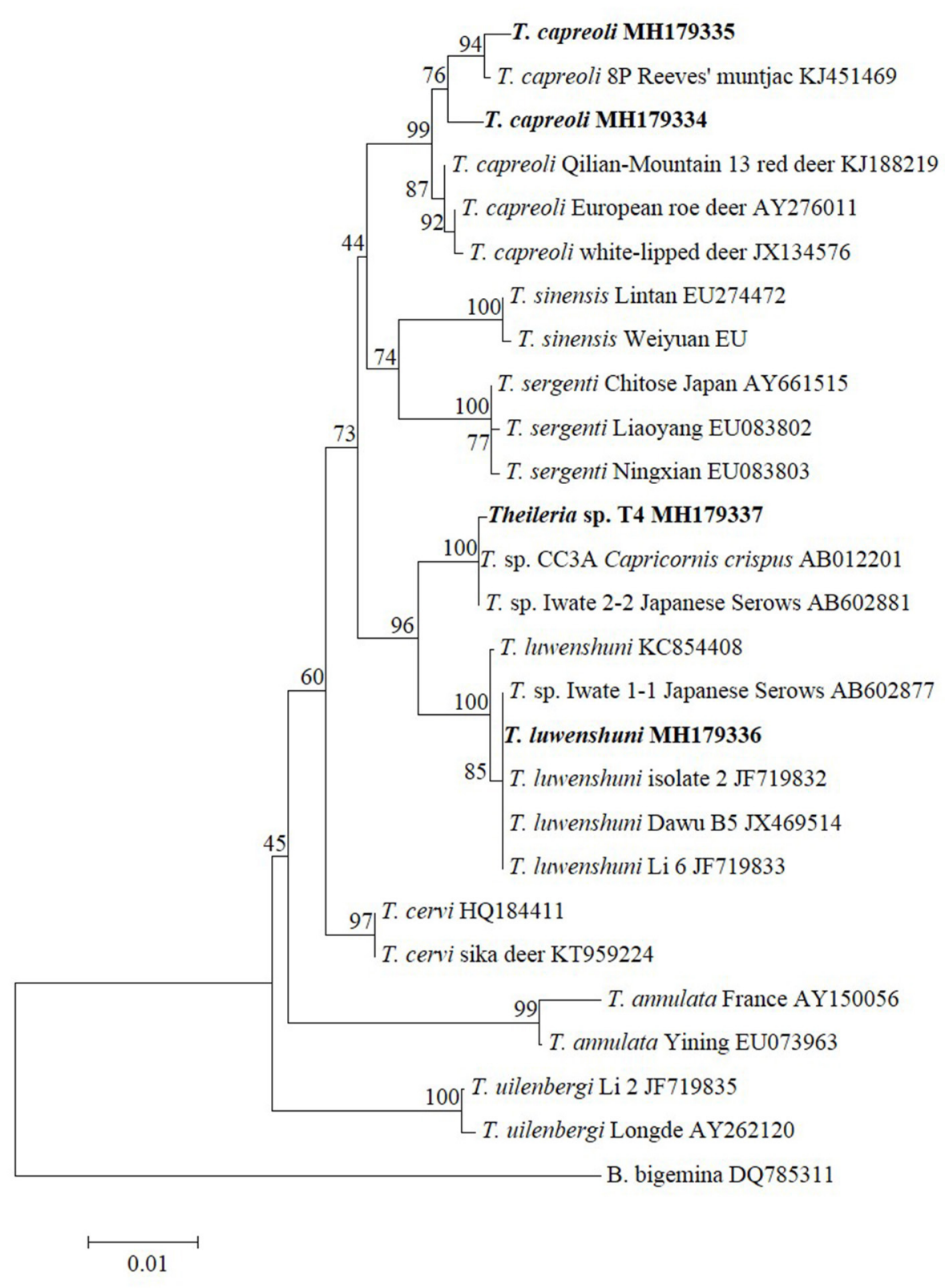

FIGURE 1 | Phylogenetic analysis of the Theileria species identified in this study based on the 18S rRNA gene. Babesia bigemina was used as outgroup. Boldface indicate the sequences obtained in this study.

humans (23). Currently, there is no evidence for transovarial transmission of $A$. phagocytophilum in tick vectors, suggesting the critical role of reservoir hosts in the maintenance and transmission of the pathogen. To date, although the occurrence of A. phagocytophilum has been widely reported in domestic animals in China (27), limited information was available in wild animals, especially wild ruminants. Several species of wild ruminants have been recognized as competent hosts for A. phagocytophilum, such as white-tailed deer in the US and roe deer and red deer in Europe (28). In the present study, A. phagocytophilum was identified in takin, Reeves' muntjac, and forest musk deer, indicating that these animals can act as a source of infections and dispersal of the agent. Previous reports have demonstrated that A. phagocytophilum could be subdivided into genetic variants based on different gene loci, which have different host tropisms and may be involved in different enzootic cycles (23). In the present study, three $16 \mathrm{~S}$ rRNA variants of $A$. phagocytophilum were obtained from those wild animals. The variants 1 and 2 identified from takin were closely related to the A. phagocytophilumlike 1 strains that have been previously found in cattle, sheep, and goats $(15,29)$. It has been suggested that $A$. 
phagocytophilum-like 1 strains are considered non-pathogenic for ruminants $(15,29)$. The variant 3 identified from Reeves' muntjac and forest musk deer was only documented in white yaks (30).

Our results first reported tick-borne pathogens in wild vertebrates that inhabit in a conservation area in southwest China. The free-ranging wild animals infected with Theileria and Anaplasma species identified herein may act as reservoir hosts for the subsequent spread of those agents. These findings provide relevant information for wildlife conservation and are also important for evaluating the risk of disease transmission between wild animals and livestock/humans.

\section{DATA AVAILABILITY STATEMENT}

The datasets presented in this study can be found in online repositories. The names of the repository/repositories and accession number(s) can be found below: https:// www.ncbi.nlm.nih.gov/genbank/, MH179333; https:// www.ncbi.nlm.nih.gov/genbank/, MH179334; https:// www.ncbi.nlm.nih.gov/genbank/, MH179335; https:// www.ncbi.nlm.nih.gov/genbank/, MH179336; https:// www.ncbi.nlm.nih.gov/genbank/, MH179337; https://www. ncbi.nlm.nih.gov/genbank/, MH180816; https://www.ncbi. nlm.nih.gov/genbank/, MH180817; https://www.ncbi.nlm. nih.gov/genbank/, MH180818; https://www.ncbi.nlm.nih. gov/genbank/, MH180819; https://www.ncbi.nlm.nih.gov/ genbank/, MH180820.

\section{REFERENCES}

1. Parola P, Davoust B, Raoult D. Tick-and flea-borne rickettsial emerging zoonoses. Vet Res. (2005) 36:469-92. doi: 10.1051/vetres:2005004

2. Diuk-Wasser MA, Vannier E, Krause PJ. Coinfection by ixodes tick-borne pathogens: ecological, epidemiological, and clinical consequences. Trends Parasitol. (2016) 32:30-42. doi: 10.1016/j.pt.2015.09.008

3. Vayssier-Taussat M, Kazimirova M, Hubalek Z, Hornok S, Farkas R, Cosson JF, et al. Emerging horizons for tick-borne pathogens: from the 'one pathogenone disease' vision to the pathobiome paradigm. Future Microbiol. (2015) 10:2033-43. doi: 10.2217/fmb.15.114

4. Orkun O, Emir H. Identification of tick-borne pathogens in ticks collected from wild animals in Turkey. Parasitol Res. (2020) 119:308391. doi: 10.1007/s00436-020-06812-2

5. Yang JF, Li YQ, Liu ZJ, Liu JL, Guan GQ, Chen Z, et al. Molecular evidence for piroplasms in wild Reeves' muntjac (Muntiacus reevesi) in China. Parasitol Int. (2014) 63:713-6. doi: 10.1016/j.parint.2014.06.002

6. Olmeda AS, Armstrong PM, Rosenthal BM, Valladares B, Delcastillo A, Dearmas F, et al. A subtropical case of human babesiosis. Acta Tropica. (1997) 67:229-34. doi: 10.1016/S0001-706X(97)00045-4

7. Barlough JE, Madigan JE, Derock E, Bigornia L. Nested polymerase chain reaction for detection of Ehrlichia equi genomic DNA in horses and ticks (Ixodes pacificus). Vet Parasitol. (1996) 63:319-29. doi: 10.1016/0304-4017(95)00904-3

8. Kawahara M, Rikihisa Y, Lin Q, Isogai E, Tahara K, Itagaki A, et al. Novel genetic variants of Anaplasma phagocytophilum, Anaplasma bovis, Anaplasma centrale, and a novel Ehrlichia sp. in wild deer and ticks on two major islands in Japan. Appl Environ Microbiol. (2006) 72:11029. doi: 10.1128/AEM.72.2.1102-1109.2006

9. Torina A, Agnone A, Blanda V, Alongi A, D'agostino R, Caracappa S, et al. Development and validation of two PCR tests for the detection of and

\section{ETHICS STATEMENT}

The animal study was reviewed and approved by Animal Ethics Committee of Lanzhou Veterinary Research Institute, Chinese Academy of Agricultural Sciences.

\section{AUTHOR CONTRIBUTIONS}

HY and JY designed this study and critically revised the manuscript. JY, XW, QN, and MM participated in sample collection and DNA preparation. JY, JW, QN, and GG performed the experiments, data analysis, and drafted the manuscript. ZL and GG participated in the coordination and manuscript revision. All authors read and approved the final manuscript.

\section{FUNDING}

This study was financially supported by the National Key Research and Development Program of China (2017YFD0501200); ASTIP, FRIP (2014ZL010), CAAS; NBCIS (CARS-37); and the Jiangsu Co-innovation Center program for Prevention and Control of Important Animal Infectious Diseases and Zoonosis.

\section{ACKNOWLEDGMENTS}

The authors would like to acknowledge the Tangjiahe National Nature Reserve for the sample collection. differentiation between Anaplasma ovis and Anaplasma marginale. Ticks Tick Borne Dis. (2012) 3:282-6. doi: 10.1016/j.ttbdis.2012.10.033

10. De La Fuente J, Atkinson MW, Naranjo V, De Mera IGF, Mangold AJ, Keating $\mathrm{KA}$, et al. Sequence analysis of the msp4 gene of Anaplasma ovis strains. Vet Microbiol. (2007) 119:375-81. doi: 10.1016/j.vetmic.2006.09.011

11. Roux V, Fournier PE, Raoult D. Differentiation of spotted fever group rickettsiae by sequencing and analysis of restriction fragment length polymorphism of PCR-amplified DNA of the gene encoding the protein rOmpA. J Clin Microbiol. (1996) 34:2058-65. doi: 10.1128/jcm.34.9.2058-2065.1996

12. Tamura K, Dudley J, Nei M, Kumar S. MEGA4: molecular evolutionary genetics analysis (MEGA) software version 4.0. Mol Biol Evol. (2007) 24:15969. doi: 10.1093/molbev/msm092

13. Li YQ, Chen Z, Liu ZJ, Liu JL, Yang JF, Li Q, et al. Molecular identification of Theileria parasites of northwestern Chinese Cervidae. Parasit Vectors. (2014) 7:225. doi: 10.1186/1756-3305-7-225

14. Li YQ, Zhang $X$, Liu ZJ, Chen Z, Yang JF, He $H N$, et al. An epidemiological survey of Theileria infections in small ruminants in central China. Vet Parasitol. (2014) 200:198-202. doi: 10.1016/j.vetpar.2013. 07.023

15. Ben Said M, Belkahia H, El Mabrouk N, Saidani M, Ben Hassen M, Alberti A, et al. Molecular typing and diagnosis of Anaplasma spp. closely related to Anaplasma phagocytophilum in ruminants from Tunisia. Ticks Tick Borne Dis. (2017) 8:412-22. doi: 10.1016/j.ttbdis.2017.01.005

16. Schreeg ME, Marr HS, Tarigo JL, Cohn LA, Bird DM, Scholl $\mathrm{EH}$, et al. Mitochondrial genome sequences and structures aid in the resolution of piroplasmida phylogeny. PLoS ONE. (2016) 11:e0165702. doi: 10.1371/journal.pone.0165702

17. Hornok S, Sugar L, Horvath G, Kovacs T, Micsutka A, Gonczi E, et al. Evidence for host specificity of Theileria capreoli genotypes in cervids. Parasit Vectors. (2017) 10:473. doi: 10.1186/s13071-017-2403-2 
18. Han JI, Jang HJ, Lee SJ, Na KJ. High prevalence of Theileria sp. in wild Chinese Water Deer (Hydropotes inermis argyropus) in South Korea. Vet Parasitol. (2009) 164:311-4. doi: 10.1016/j.vetpar.2009. 05.027

19. Yin H, Schnittger L, Luo JX, Seitzer U, Ahmed JS. Ovine theileriosis in China: a new look at an old story. Parasitol Res. (2007) 101:S1915. doi: 10.1007/s00436-007-0689-2

20. Moon KH, Sang L, Choi CY, Kim SY, Kang CW, Lee KK, et al. Investigation of Theileria sp. from Ticks and Roe Deer (Capreolus pygargus) in Jeju Island. J Vet Clin. (2014) 31:6-10. doi: 10.17555/ksvc.2014.02. 31.1.6

21. Li YQ, Liu JL, Liu ZJ, Yang JF, Li YQ, Li Q, et al. Report of Theileria luwenshuni and Theileria sp. RSR from cervids in Gansu, China. Parasitol Res. (2015) 114:2023-9. doi: 10.1007/s00436-015-4439-6

22. Dumler JS, Barbet AF, Bekker CP, Dasch GA, Palmer GH, Ray SC, et al. Reorganization of genera in the families Rickettsiaceae and Anaplasmataceae in the order Rickettsiales: unification of some species of Ehrlichia with Anaplasma, Cowdria with Ehrlichia and Ehrlichia with Neorickettsia, descriptions of six new species combin. Int J Syst Evol Microbiol. (2001) 51:2145-65. doi: 10.1099/00207713-51-6-2145

23. Battilani M, De Arcangeli S, Balboni A, Dondi F. Genetic diversity and molecular epidemiology of Anaplasma. Infect Genet Evol. (2017) 49:195211. doi: 10.1016/j.meegid.2017.01.021

24. Lee M, Yu D, Yoon J, Li Y, Lee J, Park J. Natural co-infection of Ehrlichia chaffeensis and Anaplasma bovis in a deer in South Korea. J Vet Med Sci. (2009) 71:101-3. doi: 10.1292/jvms.71.101

25. Kang JG, Ko S, Kim YJ, Yang HJ, Lee H, Shin NS, et al. New genetic variants of Anaplasma phagocytophilum and Anaplasma bovis from Korean water deer (Hydropotes inermis argyropus). Vector Borne Zoonotic Dis. (2011) 11:929-38. doi: 10.1089/vbz.2010.0214
26. Koh FX, Kai LK, Panchadcharam C, Sitam FT, Sun TT. Molecular detection of Anaplasma spp. in pangolins (Manis javanica) and wild boars (Sus scrofa) in Peninsular Malaysia. Vet Parasitol. (2016) 227:736. doi: 10.1016/j.vetpar.2016.05.025

27. Yang JF, Liu ZJ, Niu QL, Liu JL, Han R, Guan GQ, et al. Anaplasma phagocytophilum in sheep and goats in central and southeastern China. Parasit Vectors. (2016) 9:593. doi: 10.1186/s13071-016-1880-z

28. Rar V, Golovljova I. Anaplasma, Ehrlichia, and "Candidatus Neoehrlichia" bacteria: pathogenicity, biodiversity, and molecular genetic characteristics, a review. Infect Genet Evol. (2011) 11:1842-61. doi: 10.1016/j.meegid.2011.09.019

29. Aktas M, Ozubek S, Ulucesme MC. Molecular detection and phylogeny of Anaplasma phagocytophilum and related variants in small ruminants from Turkey. Animals. (2021) 11:814. doi: 10.3390/ani11030814

30. Yang JF, Liu ZJ, Niu QL, Liu JL, Guan GQ, Xie JY, et al. First molecular survey and identification of Anaplasma spp. in white yaks (Bos grunniens) in China. Parasitology. (2016) 143:686-91. doi: 10.1017/S003118201 $600041 \mathrm{X}$

Conflict of Interest: The authors declare that the research was conducted in the absence of any commercial or financial relationships that could be construed as a potential conflict of interest.

Copyright (C) 2021 Yang, Wang, Wang, Liu, Niu, Mukhtar, Guan and Yin. This is an open-access article distributed under the terms of the Creative Commons Attribution License (CC BY). The use, distribution or reproduction in other forums is permitted, provided the original author(s) and the copyright owner(s) are credited and that the original publication in this journal is cited, in accordance with accepted academic practice. No use, distribution or reproduction is permitted which does not comply with these terms. 